\title{
Plataformas virtuales en la educación superior en tiempos de COVID-19. Experiencias en estudiantes de Cuba
}

Virtual platforms in higher education in times of COVID-19. Experiences in Cuban students

\section{Volumen 21, Número 3 \\ Setiembre - Diciembre \\ pp. $1-20$}

\author{
Elizabeth Jiménez-Puig \\ Zoylen Fernández-Fleites
}

Citar este documento según modelo APA

Jiménez-Puig, Elizabeth. y Fernández-Fleites, Zoylen. (2021). Plataformas virtuales en la educación superior en tiempos de COVID-19. Experiencias en estudiantes de Cuba. Revista Actualidades Investigativas en Educación, 21(3), 1-20. Doi. 10.15517/aie.v21i3.46224 


\title{
Plataformas virtuales en la educación superior en tiempos de COVID-19. Experiencias en estudiantes de Cuba
}

Title: Virtual platforms in higher education in times of COVID-19. Experiences in Cuban students

\author{
Elizabeth Jiménez-Puig ${ }^{1}$ \\ Zoylen Fernández-Fleites ${ }^{2}$
}

Resumen: En la actualidad, las universidades han cerrado sus puertas y se ha implementado un proceso educativo basado en el aprendizaje en línea. El presente artículo expone los resultados de un estudio cuyo principal objetivo fue explorar las preferencias del alumnado de Psicología de la Universidad Central "Marta Abreu" de Las Villas, en relación con el empleo de plataformas virtuales dentro del proceso docente de educación a distancia. Se desarrolló una investigación cuantitativa, con un diseño transversal de tipo descriptivo, mediante cuestionario, con alcance descriptivo, en febrero de 2021. Se empleó un cuestionario a través del software libre Google Forms ${ }^{\circledR}$. Se realizó un muestreo no probabilístico e intencional. Un total de 72 estudiantes completó el cuestionario. Los datos fueron procesados usando SPSS (Statistical Package for Social Sciences), versión 25, mediante el empleo de la estadística descriptiva. Los resultados mostraron que del grupo de plataformas disponibles para el proceso de educación en línea, la mayoría del alumnado emplea WhatsApp alegando que resulta frecuente su uso entre colegas de estudio, que se encuentran familiarizados con su funcionamiento y que facilita la comunicación. La segunda opción de elección fue el correo personal, y, en tercer lugar, la plataforma Moodle. Conocer las preferencias y motivaciones del alumnado, para dar respuestas a sus quehaceres académicos, orienta al profesorado y la dirección en cuanto al reconocimiento de diversas plataformas, y no solamente de aquellas dictadas institucionalmente. Por tanto, debe hacerse un llamado a la flexibilidad, a la diversidad de posibilidades, un ajuste necesario a lo que el alumnado considera motivante y atrayente.

Palabras clave: educación a distancia, universidad, aislamiento social, aprendizaje en línea.

Abstract: Currently, universities have closed their doors and an educational process based on online learning has been implemented. This article presents the results of a study whose main objective was to explore the preferences of the Psychology students of the Central University "Marta Abreu" of Las Villas, in relation to the use of virtual platforms within the distance education teaching process. A quantitative research was carried out, with a descriptive cross-sectional design using a questionnaire, with a descriptive scope, in February 2021. A questionnaire was used through the free software Google Forms ${ }^{\circledR}$. A non-probabilistic and intentional sampling was carried out. A total of 72 students completed the questionnaire. The data were processed using SPSS / Windows, version 25, using descriptive statisTic. The results showed that of the group of platforms available for the online education process, most of the students use WhatsApp, claiming that its use is frequent among study colleagues; who are familiar with how it works; and that facilitates communication. The second option of choice was personal email, and third, the Moodle platform. Knowing the preferences and motivations of students to give answers to their academic tasks, guides teachers and managers regarding the recognition of various platforms, and not only those dictated institutionally. At present, a call must be made for flexibility, for diversity of possibilities; a necessary adjustment to what the students consider motivating and attractive.

Keywords: distance education, university, social alienation, electronic learning.

\footnotetext{
${ }_{1}$ Profesora Asistente de la Universidad Central "Marta Abreu" de Las Villas, Santa Clara, Cuba. Máster en Psicopedagogía. Dirección electrónica: ejimenez@uclv.cu Orcid https://orcid.org/0000-0002-7683-6096
}

2 Profesora Instructora de la Universidad Central "Marta Abreu" de Las Villas, Santa Clara, Cuba. Máster en Psicología Médica. Dirección electrónica: zfernandez@uclv.cu Orcid https://orcid.org/0000-0001-9739-2749

Artículo recibido: 16 de marzo, 2021

Enviado a corrección: 21 de junio, 2021

Aprobado: 16 de agosto, 2021 


\section{Introducción}

En 2019, la provincia de Hubei, China, se convirtió en el epicentro del nuevo brote de coronavirus. Se trataba del SARS-CoV2, causante de la enfermedad del coronavirus 2019 o COVID-19, tipificada así por la Organización Mundial de la Salud (OMS). Actualmente, es considerada una emergencia sanitaria mundial. El cuadro clínico de la COVID-19 varía desde una sintomatología leve y autolimitante del tracto respiratorio hasta una neumonía progresiva grave, insuficiencia multiorgánica y muerte. Hasta la fecha no existen agentes terapéuticos específicos para las infecciones por SARS-CoV-2 (Aquino-Canchari y Medina-Quispe, 2020).

Una de las medidas para disminuir la propagación de la COVID-19 es el aislamiento social y la inmovilización social obligatoria. La necesidad y urgencia que trajo consigo la crisis sanitaria actual hizo que los gobiernos cerraran las puertas de las instituciones educativas como una medida para mitigar los efectos de la pandemia (Aquino-Canchari y Medina-Quispe, 2020). Según la UNESCO (2020), más de 138 países mantienen totalmente cerrado su sistema educativo, lo cual afecta al $94 \%$ del alumnado a nivel mundial. Esta realidad ha provocado, en el mundo entero, y en Latinoamérica, en particular, en el ámbito de la educación superior, una situación compleja y atípica en todos los niveles (Cabero-Almenara y LlorenteCejudo, 2020). Ante esta problemática, las universidades han optado por la educación a distancia y el aprendizaje en línea como respuesta a la COVID-19, para así adecuarse a esta situación absolutamente excepcional y no dejar de atender a la población estudiantil (AquinoCanchari y Medina-Quispe, 2020).

En momentos de crisis, de aislamiento social y de confinamiento, pasar de la presencialidad en educación a la virtualidad ha significado un nuevo reto y desafío para la mayoría de las instituciones de educación superior. El desafío no es solo desde el punto de vista del proceso de enseñanza y aprendizaje, sino también de las brechas a nivel de oportunidades de accesibilidad y tecnología que tiene el alumnado para conectarse a la dinámica del aprendizaje en línea (Canales y Silva, 2020).

Cuba no se encuentra exenta de esta realidad. El alumnado universitario cubano se enfrenta a un proceso de educación a distancia basado en plataformas virtuales, con algunas dificultades de conectividad y acceso a dispositivos electrónicos. En Cuba, fueron suspendidas las actividades docentes, en la Educación Superior, por primera vez, a raíz de la COVID-19, el 23 de marzo de 2020, con la expectativa de retomar pasadas 3 semanas (Alonso, Figueredo, Fuentes, Doimeadiós, y otros, 2020). Debido al avance de la pandemia en el territorio se 
ofrecieron nuevas medidas, esto al no poderse cumplir dicha expectativa y continuar el aislamiento social (Alonso, Figueredo, Fuentes, Izquierdo, y otros, 2020).

En la Universidad Central "Marta Abreu" de Las Villas (UCLV), las actividades docentes reiniciaron en septiembre de 2020, lo cual permitió a docentes y estudiantes la culminación del curso escolar y la realización de exámenes (Hernandez, 2020). Las actividades docentes correspondientes al curso 2021 se retomaron el pasado 2 de marzo de 2021 (Sol, 2021).

Si bien, desde el primer confinamiento se había orientado el uso de las plataformas virtuales, en este reinicio del curso 2021, se incorpora un bloque de docencia completamente a distancia, en el cual el alumnado debe no solo desarrollar sus habilidades en cada una de las materias, sino también en la gestión de la información en el aprendizaje en línea mediante el empleo de las aulas y plataformas virtuales. La UCLV pone a disposición de sus estudiantes la plataforma virtual Moodle. No obstante, el alumnado encuentra otras vías de comunicación con colegas y con el profesorado, que no se circunscriben a esta plataforma. Precisamente por ello, la presente investigación persiguió como objetivo: explorar las preferencias del alumnado de Piscología de la UCLV en relación con el empleo de plataformas virtuales dentro del proceso de educación a distancia. La investigación se llevó a cabo durante el mes de febrero de 2021.

\section{Referentes teóricos}

La importancia que ha adquirido el aprendizaje en línea, en el 2020, resulta un hecho sin precedentes que marcará un antes y un después en las prácticas pedagógicas y en los sistemas educativos actuales a nivel global (Expósito y Marsollier, 2020). La implementación de la educación a distancia, a través de aulas virtuales, representa un desafío para las autoridades universitarias, docentes y estudiantes debido a ciertas consideraciones necesarias para su adecuada aplicación. Se trata de la disponibilidad de internet, un ambiente adecuado para estudiar en casa, el manejo de plataformas virtuales por parte de docentes y estudiantes, capacidad de la persona docente para dictar cursos a distancia, así como la existencia de un soporte académico tecnológico de la universidad (Aquino-Canchari y MedinaQuispe, 2020).

Por ende, la educación a distancia se ha de convertir en una herramienta social para transformar el comportamiento de los ciudadanos desde este momento fuera del aula, principalmente en sus hogares. El cambio de paradigma comienza con el aumento del uso de herramientas tecnológicas: aplicaciones, plataformas digitales mediante el uso de aulas 
virtuales, dispositivos y softwares que permiten la comunicación de manera interactiva, a pesar del distanciamiento social existente provocado por esta pandemia (Arce-Peralta, 2020).

Si bien, desde hace algunos años el empleo de las Tecnologías de la Información y Comunicación (TIC) ha estado incorporado a los currículos de diversas carreras, en esta situación actual, se convierten en protagonistas para la facilitación de los procesos de educación a distancia en las universidades. De este modo, se reconoce que la incorporación de las Tic en la vida cotidiana en la sociedad ha propiciado la aparición y uso de herramientas que permiten a las personas comunicarse y organizarse. Varios autores han hecho referencia al término ciudadanía digital para describir un cúmulo de habilidades que deben poseer los ciudadanos para la navegación en línea, especialmente, el alumnado y el profesorado universitario, para enriquecer la dinámica de los procesos de enseñanza (Torres, 2018). La situación generada por la COVID-19 ha puesto de manifiesto la imperiosa necesidad de realizar este tránsito a través de la imposición de su realización no de forma procesual, sino de manera instantánea.

De esta manera, se recurre a un proceso de aprendizaje en línea, y las clases virtuales adquieren un rol protagónico. La clase virtual es entendida como un espacio de alguna plataforma online, en la cual docentes y estudiantes comparten información, y en la que se atienden dudas, consultas y se puede evaluar a quienes participan. La condición indispensable para acceder a ellas es el acceso a Internet y una computadora o celular inteligente (Aguirre, Zhindon y Pomaquero, 2020). Este tipo de aprendizaje en línea ha mostrado excelentes resultados en revisiones realizadas; se han encontrado cifras de impacto similares a la modalidad presencial. No obstante, Aquino-Canchari y Medina-Quispe (2020) apuntan que dichas investigaciones no fueron realizadas en la actual situación epidemiológica de aislamiento social y confinamiento.

A la actual realidad ha venido aparejado un sinnúmero de dificultades para el acceso a las plataformas virtuales. Existen, incluso, regiones en las cuales las universidades no cuentan con este recurso, como es el caso de Ecuador (Aguirre y Zhindon, 2020). En la investigación realizada en la Universidad San Francisco de Paula Santander Ocaña, Colombia, el alumnado reportó dificultades para mantenerse conectado todo el día (Arellano, 2021). Por otra parte, en Argentina, se encontró que el profesorado presenta disímiles dificultades tecnológicas, existe sobrecarga en las plataformas debido al flujo de acceso y la posesión de teléfonos compartidos no personales (Brítez, 2020). En el caso de Paraguay, de las 9 universidades públicas y de las 46 privadas existentes, solamente 10 cuentan con plataformas para clases virtuales (Brítez, 
2020). En el caso de la Pontificia Universidad Católica de Perú, se obtuvieron resultados que indicaron una posible deserción escolar en estudiantes con bajos recursos económicos y dificultades de acceso a la virtualidad (Lovón y Cisneros, 2020).

En Cuba, la realidad de la conectividad no dista considerablemente de las anteriores. Las personas deben abonar pagos de Internet, a razón de 100 pesos cubanos por cada GigaBite (GB). Este precio va aumentando mientras más GB desee adquirir el usuario. No todo el alumnado cuenta con los dispositivos que permiten y facilitan la conexión, además de que esta no resulta ideal. De manera general, se ha reportado que el alumnado latinoamericano, con respecto al resto de estudiantes a nivel mundial, perciben preocupaciones actuales vinculadas con el equipamiento del cual disponen para el uso de las TIC, por la conexión a Internet y su calidad, por las dificultades para comunicarse con sus profesores y por la situación económica de sus países y hogares (Cabero-Almenara y Llorente-Cejudo, 2020).

No obstante, la educación a distancia y el aprendizaje en línea constituyen una realidad innegable en la universidad en los momentos actuales. En numerosos estudios, pese a las dificultades con las cuales se enfrentan docentes y estudiantes, han surgido propuestas interesantes para el acercamiento a esta forma de enseñanza. De este modo, en México, las principales vías para la virtualidad en los espacios universitarios la constituyen las carpetas digitales en Google Drive, los grupos de WhatsApp, las plataformas ZOOM, Google Meet, Microsoft Team, así como programas de radio y Podcats (Arce-Peralta, 2020). En el estudio conducido por Arellano (2021), en la Escuela Normal Superior del Valle de México, el alumnado alegó que para la comunicación con docentes y colegas, así como la realización de tareas, emplean el aula virtual, el correo electrónico y el WhatsApp. También en Cuba, en estudiantes de Ciencias Médicas, se han puesto de manifiesto las bondades del WhatsApp en el envío de imágenes, tan necesarias en muchas de las materias que se imparten en estas ramas de las ciencias (Esquivel, Martínez-Fortún, Águila, y Llerena, 2020).

La UCLV posee la propuesta del aula virtual mediante el empleo de la plataforma Moodle (Modular Object Oriented Dynamic Environment). En ella se encuentran ubicados todos los materiales referentes a asignaturas, cursos, carreras y años académicos. La universidad provee un sistema de cuentas de usuario a cada estudiante mediante las cuales pueden acceder a cada una de sus clases virtuales. Debido a las dificultades de conexión se han gestionado un cúmulo de opciones para facilitar el acceso libre de costo hacia el Portal Web Universitario, el Campus Virtual Moodle, el servicio de correo UCLV y la nube UCLV. Estas opciones son libres de costo siempre y cuando la conexión se realice desde zonas con acceso 
Wi-Fi o desde el NautaHogar (UCLV, 2021). El acceso desde otras modalidades no resulta libre de costos para el profesorado ni para el estudiantado.

Para el alumnado de la UCLV se añade otra problemática, la gestión de sus conocimientos debe hacerse mediante el empleo de la plataforma Moodle. Allí y solamente allí podrán ser evaluados y se registrará su matrícula y participación. No obstante, esta plataforma es considerada como una Plataforma de Apoyo a la Presencialidad (Batara y Rapat, 2020). Los resultados preliminares de su aplicación en la Universidad San Francisco de Paula Santander Ocaña, Colombia, indicaron que Moodle por sí solo es un apoyo al proceso formativo desescolarizado, mas no brinda un proceso de formación $100 \%$ virtual. Herramientas, tecnología y estrategias adicionales deben ser usadas como complemento, algunas de ellas serían: Ilamadas telefónicas (estudiantes y familiares), correo institucional y WhatsApp (llamada y mensajes de texto). Por su parte, para motivar la participación con grandes resultados, Google Meet, etc. (Areniz-Arevalo y otros, 2020).

\section{Metodología}

\subsection{Enfoque}

Se desarrolló una investigación cuantitativa con un diseño transversal de tipo descriptivo mediante cuestionario, con alcance descriptivo (León y Montero, 2015). En este tipo de estudios, se busca describir una determinada población en un momento dado a través del sondeo de opiniones con el uso de cuestionarios. Los instrumentos pueden estar ya estandarizados para su empleo, o puede tratarse de cuestionarios creados por quienes investigan para conocer el estado actual de determinada cuestión. El cuestionario debe responder a los objetivos trazados en el estudio, y puede ser aplicado sin la presencia de las personas que evalúan.

\subsection{Unidades de análisis}

La población estuvo constituida por 115 estudiantes continuantes de la carrera Licenciatura en Psicología de la Universidad Central "Marta Abreu" de Las Villas, Cuba. Se realizó un muestreo no probabilístico e intencional, a partir del establecimiento de criterios de selección muestral:

Criterios de inclusión:

- Ser estudiante continuante de Licenciatura en Psicología en la Universidad Central "Marta Abreu" de Las Villas.

- Ofrecer su consentimiento para participar en la investigación. 
Criterio de exclusión:

- Presentar dificultades tecnológicas y/o de conectividad que impidiesen ofrecer respuesta al instrumento de recopilación de información.

Criterios de salida:

- Respuestas incompletas.

- Abandono voluntario.

Un total de 72 estudiantes completó el cuestionario, cifra que representa el $62.61 \%$ de la población. Los datos correspondientes a las personas participantes en la investigación se muestran a continuación en la Tabla 1.

Tabla 1

Psicología UCLV: Frecuencia y porcentaje de las características de la muestra según edad, sexo, año académico, provincia y zona de residencia (febrero de 2021, Cuba)

\begin{tabular}{|c|c|c|}
\hline & ariables & Frecuencia (\%) \\
\hline Media edad & & 21.1(1.6) \\
\hline Sexo & Mujer & $63(87.5)$ \\
\hline & Hombre & $9(12.5)$ \\
\hline Año & Segundo & $23(31.9)$ \\
\hline & Tercero & $17(23.6)$ \\
\hline & Cuarto & $19(26.4)$ \\
\hline & Quinto & $13(18.1)$ \\
\hline Provincia & Villa Clara & $27(37.5)$ \\
\hline & Ciego de Ávila & $11(15.3)$ \\
\hline & Camagüey & $11(15.3)$ \\
\hline & Cienfuegos & $15(20.8)$ \\
\hline & Sancti Spíritus & $8(11.1)$ \\
\hline Zona de & Urbana & $59(81.9)$ \\
\hline Residencia & Rural & $13(18.1)$ \\
\hline
\end{tabular}

Fuente: Elaboración propia sobre los datos aportados por la Encuesta sobre empleo de plataformas virtuales. Primera parte

\subsection{Técnicas de recolección}

La encuesta fue anónima y confidencial. Los datos de las personas participantes fueron recolectados en el mes de febrero de 2021 empleando un cuestionario de aplicación mediante internet. La investigación se desarrolló con el empleo del software libre Google Forms®. Se obtuvo el consentimiento online del estudiantado. Se empleó un cuestionario diseñado para la 
investigación, constituido por un primer bloque de preguntas sobre datos generales (sexo, edad, año académico, provincia y zona de residencia); en un segundo bloque, se incluyeron un total de cuatro preguntas dirigidas hacia la descripción de las preferencias en empleo de plataformas virtuales en el marco de la educación en línea. La primera pregunta está orientada a identificar las plataformas online empleadas (de las posibles a seleccionar: WhatsApp, Telegram, Moodle, Sijú, Correo UCLV, Correo personal, Nube personal, Nube UCLV). La segunda pregunta solicitaba el establecimiento de jerarquía entre las plataformas seleccionadas en función de las que mayor uso poseían para las personas participantes. La tercera pregunta indaga sobre el motivo de la preferencia en relación con el empleo de una plataforma. Por último, la cuarta pregunta solicita información sobre el porqué de la ubicación de una plataforma determinada en última posición (Anexo 1).

\subsection{Procesamiento de análisis}

Los datos fueron procesados usando SPSS (Statistical Package for Social Sciences) Windows, versión 25. Se empleó la estadística descriptiva para explorar las características de las personas participantes, así como la opinión acerca del empleo de plataformas online.

\section{Resultados y discusión}

Del grupo de plataformas disponibles para el proceso de educación a distancia, la mayoría emplea WhatsApp (98.9 \%). En el caso del correo personal, es empleado por el $63.9 \%$ de las personas participantes, un $23 \%$ emplea la plataforma Moodle y un $26.4 \%$, Telegram. Por otra parte, el acceso tanto a la nube personal como a la prestablecida desde la Institución mostró una frecuencia menor de selección en las personas participantes (5.6 \% cada una) (Tabla 2). 
Tabla 2

Psicología UCLV: Frecuencia y porcentaje de las plataformas online empleadas por el estudiantado en la resolución de demandas

\begin{tabular}{|c|c|}
\hline Plataforma & Frecuencia (\%) \\
\hline WhatsApp & 71(98.9) \\
\hline Correo personal & $46(63.9)$ \\
\hline Moodle & $23(31.9)$ \\
\hline Telegram & $19(26.4)$ \\
\hline Correo UCLV & $13(18.1)$ \\
\hline Sijú & $7(9.7)$ \\
\hline Nube personal & $4(5.6)$ \\
\hline Nube UCLV & $4(5.6)$ \\
\hline
\end{tabular}

Fuente: Elaboración propia sobre los datos aportados por la Encuesta sobre empleo de plataformas virtuales

En cuanto a las preferencias para el empleo de una u otra plataforma, el $88.9 \%$ emplea WhatsApp como plataforma principal para el aprendizaje en línea. El resto de las plataformas constituye la opción principal para una frecuencia mínima de estudiantes, que oscila entre 1.4 $\%$ y $2.8 \%$, en función de la plataforma puntual (Tabla 3 ).

Tabla 3

Psicología UCLV: Frecuencia y porcentaje de plataformas online de elección por parte del estudiantado en la realización de demandas académicas (ubicada en primer lugar) (febrero, 2021, Cuba)

\begin{tabular}{|l|l|}
\hline Plataforma & Frecuencia (\%) \\
\hline WhatsApp & $64(88.9)$ \\
\hline Nube UCLV* & $3(4.2)$ \\
\hline Correo personal & $2(2.8)$ \\
\hline Nube personal & $2(2.8)$ \\
\hline Telegram & $1(1.4)$ \\
\hline Moodle & $1(1.4)$ \\
\hline Sijú & $1(1.4)$ \\
\hline Correo UCLV & $1(1.4)$ \\
\hline
\end{tabular}

Nota. *ningún estudiante lo seleccionó en primer lugar, el porcentaje reflejado hace referencia al tercer lugar

Fuente: Elaboración propia sobre los datos aportados por la Encuesta sobre empleo de plataformas virtuales

Dentro de las causas que motivan ubicar una plataforma en primer lugar, el $88.9 \%$ expresó que resulta frecuente su uso entre colegas de estudio. El $62.5 \%$ se encuentra familiarizado con su funcionamiento, y el 58.3 \% coincidió en que facilita la comunicación. 
Tabla 4

Psicología UCLV: Frecuencia y porcentaje ante la consulta ¿Por qué la plataforma ubicada en primer lugar es su preferida en la realización de demandas académicas? (febrero, 2021, Cuba)

\begin{tabular}{|l|l|}
\hline Declaración & Frecuenc|a \\
\hline $\begin{array}{l}\text { Resulta muy frecuente su uso entre mis colegas de } \\
\text { estudio, por lo que hay más posibilidades de que estén } \\
\text { en línea cuando los necesito. }\end{array}$ & $64(88.9)$ \\
\hline Me encuentro familiarizado (a) con su funcionamiento. & $45(62.5)$ \\
\hline Facilita la comunicación. & $42(58.3)$ \\
\hline Me resulta atractiva. & $12(16.7)$ \\
\hline
\end{tabular}

Fuente: Elaboración propia sobre los datos aportados por la Encuesta sobre empleo de plataformas virtuales

Con relación a las causas que motivaron ubicar una plataforma en último lugar de preferencia, el 35 \% aseguró que las personas con las que comparte no emplean la plataforma, mientras que la dificultad para enviar archivos grandes o múltiples constituyen un motivo para que el $30.6 \%$ desplace hacia el final una plataforma online.

\section{Tabla 5}

Psicología UCLV: Frecuencia y porcentaje ante la consulta ¿Por qué la plataforma ubicada al final es la menos preferida por Ud. en la realización de demandas académicas? (febrero, 2021, Cuba)

\begin{tabular}{|l|l|}
\hline Declaración & Frecuencia (\%) \\
\hline $\begin{array}{l}\text { La mayoría de las personas con quienes comparto para } \\
\text { tareas académicas no la usan. }\end{array}$ & $35(48.6)$ \\
\hline $\begin{array}{l}\text { Se dificulta el envío de archivos grandes y/o archivos } \\
\text { múltiples. }\end{array}$ & $22(30.6)$ \\
\hline No me encuentro familiarizado (a) con su funcionamiento. & $20(27.8)$ \\
\hline No resulta de fácil acceso. & $18(25.0)$ \\
\hline Se demora en cargar, es lenta. & $17(23.6)$ \\
\hline No me atrae. & $12(16.7)$ \\
\hline
\end{tabular}

Fuente: Encuesta sobre empleo de plataformas virtuales

Los resultados reportados son susceptibles de dos análisis esenciales: por una parte, las plataformas que emplea el alumnado para sus tareas académicas, en su proceso de aprendizaje en línea, y, por otro lado, el análisis de aquellas que resultan de su preferencia. Se realiza esta salvedad, pues, si bien el alumnado puede indicar las plataformas que utiliza para la realización de sus tareas, esto no necesariamente significa que es la que prefieran para ello.

En este sentido, el estudiantado continuante de la carrera Licenciatura en Psicología de la UCLV utiliza con mayor frecuencia el WhatsApp, el correo personal y el Moodle para ofrecer 
respuestas a sus demandas académicas. No obstante, cuando se trata de sus preferencias eligen la plataforma de comunicación WhatsApp alegando razones referidas a que les facilita la comunicación, es común su empleo entre los colegas de estudio, se encuentran familiarizados con ella, etc. Este hecho no resulta extraño, ya que, alrededor de todo el mundo, e incluso, en Cuba, esta aplicación se ha convertido en la opción de comunicación elegida por muchos en las actuales condiciones de aislamiento social. Además, entre sus bondades se ha destacado la posibilidad de aclarar dudas, permitir la retroalimentación de forma rápida, así como tener acceso a materiales formativos en diversos formatos (Suárez, 2018).

Estudios conducidos en España han mostrado la importancia que el alumnado universitario le confiere al WhatsApp en la realización de sus tareas escolares (FondevilaGascón, Marqués-Pascual, Mir-Bernal, y Polo-López, 2019), no solamente circunscritos a la situación actual, sino que, desde hace varios años, se vienen investigando las bondades que ofrece la aplicación en la comunicación y creación de grupos de estudio. Se han encontrado mejoras en las competencias comunicativas y participativas de estudiantes vinculados a estos grupos (Pérez-Jorge y otros, 2018). En estudios conducidos en México, previo al confinamiento y cierre de los centros universitarios ya se apuntaban las preferencias del alumnado por esta red comunicacional alegando una mejora del $95 \%$ en las interacciones docente- estudiante y estudiante- docente (Hernández, Raya, y Santana, 2017). En adición, Trejos (2018) apuntaba acerca de la preferencia del estudiantado universitario hacia el WhatsApp, gracias a la facilidad que ofrece en la comunicación con profesores.

Estas preferencias y la utilidad del WhatsApp se han amplificado en las condiciones actuales de enseñanza. Tal fue el resultado aportado por Susilawati y Supriyatno (2020) en la investigación conducida con 30 estudiantes del Departamento del Estado Islámico de la Universidad de Maluana Ibrahim Malang. De igual forma, 30 estudiantes y 24 docentes pertenecientes a las 9 universidades públicas de Egipto prefirieron el Facebook y el WhatsApp para la orientación y realización de actividades docentes (Sobaih, Hasanein, y Abu, 2020). En este sentido Yudha, Luthfi, y Prayudi (2017) apuntan que el WhatsApp constituye una aplicación versátil y multiplataforma.

Incluso, en el contexto cubano, han existido evidencias del impacto de redes sociales en actividades académicas, que no se reducen a las aulas virtuales. Un ejemplo de ello lo constituye la experiencia de la Universidad de Holguín, donde se llevó a cabo el proceso de graduación de 45 estudiantes culminantes mediante el empleo de redes como Facebook, 
Messenger, WhatsApp, Telegram, ToDus y Sijú, todas con excelentes resultados y cumplimiento de objetivos (Vega, Pupo, Tapia, y Pérez, 2021).

El uso del correo electrónico también continúa siendo una preferencia del alumnado y del profesorado en cuanto a comunicación, tal como lo señalaba Arellano (2021) en su investigación acerca de la implementación de aulas virtuales en México. En Cuba, el correo electrónico sigue siendo una fuente importante de transmisión de información, y el alumnado prefiere el empleo de sus correos personales, no así, el ofrecido por la institución. Coherente con ello, en el estudio de Macchiarola, Pizzolitto, Pugliese, y Muñoz (2020), en 6250 estudiantes de la Universidad Nacional de Río Cuarto (UNRC) en Argentina, se encontró que las herramientas tecnológicas más utilizadas en las clases virtuales son el correo electrónico y las plataformas institucionales.

Por otra parte, también existen evidencias del empleo de la plataforma Moodle como herramienta para la educación a distancia. En la Universidad de Ciencias Médicas de Santiago de Cuba, se condujo un estudio descriptivo-transversal acerca del empleo del aula virtual. En sus resultados, pese a que una gran cantidad de estudiantes conocían cómo acceder y habían tenido algún contacto con esta, otro tanto nunca lo había hecho, y carecían de conocimientos para acceder. En adición, un elevado porcentaje de los 150 participantes reportaron insatisfacciones en cuanto a la comunicación con profesores y colegas, así como con el proceso de evaluación (Callís y otros, 2020). Este elemento se corresponde con lo encontrado acerca de las bondades comunicativas que el alumnado encuentra en WhatsApp, no así en el aula virtual.

En la Universidad Autónoma del Estado de Morelos, México, se desarrolló un estudio descriptivo y correlacional en el cual participaron 425 estudiantes. En la misma, los autores encontraron que el 72.5 \% usan la plataforma institucionalizada, que no es el Moodle. Sin embargo, el Moodle lo usa el 17.2 \% de las personas participantes (Delgado y Martínez, 2021). Del mismo modo sucedió en la Facultad de Odontología de la Universidad Nacional Mayor de San Marcos, Perú. De 188 estudiantes participantes en la investigación, el $11.2 \%$ utilizan el Moodle, mientras que el 88.8 \% prefieren otras plataformas en función del aprendizaje en línea (Mezarina, Evaristo, Ortiz, y Usca, 2020).

Por otra parte, en la Universidad de Saida, Alergia, se investigaron las preferencias en estudiantes y profesores. La plataforma preferida resultó ser Facebook, seguida de Moodle. No obstante, resulta necesario aclarar que la elección del Moodle se realizó haciendo la salvedad de su uso como plataforma de apoyo a otras formas de comunicación online 
(Ghounane, 2020). Y es que, ha sido señalado que la plataforma Moodle por parte de los profesores, se emplea como repositorio de materiales de apoyo y para la recolección de informes del estudiantado (Del Prete, Cabero, y Halal, 2018).

Resultados similares a estos fueron obtenidos en 414 estudiantes de las Carreras de Psicología y Ciencias de la Educación, de la Universidad Mayor de San Andrés, Colombia. En esta institución, se ha ampliado en un $22.9 \%$ el uso del Moodle como plataforma institucional para el aprendizaje a distancia. No obstante, se reconoció que las plataformas no son usadas como debería ser, ya que, tan solo se envía información y las evaluaciones son tradicionales (Ordoñez, 2020). La investigación realizada en la Universidad de Extremadura, España, con 548 estudiantes, aportó datos de interés que indicaron que las clases virtuales han consistido esencialmente en presentaciones subidas al campus virtual con interacciones asíncronas. La valoración negativa que hacen de la enseñanza a distancia se explica por la inversa relación percibida entre dedicación al estudio y rendimiento académico (Pérez-López, Vázquez, y Cambero, 2020).

Aun cuando en el presente estudio se haya referido el uso del aula virtual en Moodle, pero no su preferencia, es necesario destacar que esta forma de enseñanza online tiene beneficios tanto para profesores como para estudiantes. A decir de Cortés, Cortés, Medina, Manzano, y León (2020), se trata de una plataforma que siempre está disponible para todos las 24 horas del día, de modo que, si el alumnado no puede revisar el contenido que el profesorado ha dejado para el estudio, podrá revisarlo en cualquier momento. El profesorado puede tener el control de la asistencia gracias a las herramientas de monitoreo y control y se puede conocer cuando el estudiantado accede al contenido o si lo descarga. No obstante, se ha planteado que la cantidad de estudiantes que acceden a la educación a distancia desde la plataforma Moodle, depende de las condiciones técnicas de cada cual (Bravo, Larrea, Ruales, y Cruz, 2020).

De forma general, las investigaciones acerca de la educación a distancia mediante el aprendizaje en línea, advierten dificultades de conectividad, déficits en cuanto a disponibilidad de dispositivos para la realización del aprendizaje en línea (muchas veces, se cuenta solamente con un teléfono móvil, lo cual entorpece la lectura de textos y redacción de documentos). Además, se ha planteado que el estudiantado no advierte una propuesta coherente e integral de educación virtual en su carrera (Macchiarola y otros, 2020). Precisamente por ello, Pérez-López y otros (2020) aseveran que la universidad debe transitar hacia modelos más colaborativos y centrados en el estudiante. 


\section{Conclusiones}

La investigación desarrollada constituyó un acercamiento preliminar a aquellas herramientas digitales que emplea y prefiere el alumnado de la Carrera de Licenciatura en Psicología de la Universidad Central "Marta Abreu" de Las Villas. El estudio permitió el conocimiento de sus motivaciones e intereses, así como las razones por las cuales seleccionaron una u otra vía online para la realización de sus tareas académicas. Conocer las preferencias y motivaciones del alumnado para dar respuestas a sus quehaceres académicos orienta a la dirección de las instituciones en cuanto a estrategias para el reconocimiento de diversas plataformas, y no solamente de aquellas dictadas institucionalmente.

Se obtuvo que la mayoría del alumnado de la Carrera de Licenciatura en Psicología de la Universidad Central "Marta Abreu" de Las Villas emplea WhatsApp como plataforma de elección para las demandas de la educación a distancia. El estudiantado refirió que resulta frecuente su uso entre colegas de estudio, que se encuentran familiarizados con su funcionamiento y que facilita la comunicación. La segunda opción de elección fue el correo personal y, en tercer lugar, la plataforma institucional Moodle.

Si bien, el Moodle constituye una opción institucional que ofrece disímiles ventajas a estudiantes y profesores no debe constituir la única vía reconocida para la gestión de tareas, clases y evaluaciones. En la actualidad, debe hacerse un llamado a la flexibilidad, a la diversidad de posibilidades, un ajuste necesario a lo que el alumnado considera motivante y atrayente.

Precisamente por ello convendría la realización de investigaciones similares que pudiesen profundizar en cuestiones como: ¿A qué se debe que el estudiantado de la carrera de Psicología de la UCLV prefiera otras plataformas para el aprendizaje en línea si la institución le facilita una plataforma? ¿Sucede de este mismo modo con el resto del alumnado de otras carreras? ¿Podrían llegar a reconocerse de manera institucional estas otras plataformas que el estudiantado está empleando y que da cuenta de su labor en la educación a distancia?

Las autoras, aun cuando consideran la valía de los resultados obtenidos, también reconocen la existencia de limitaciones en la investigación, esencialmente, el tamaño de la muestra, que dificultó la realización de otros niveles de análisis. No obstante, al constituir un primer acercamiento deja una brecha para futuras investigaciones; por ejemplo, la extensión del estudio hacia toda la comunidad universitaria y enriquecer los datos con aportes desde un enfoque cualitativo, etc. 


\section{Referencias}

Aguirre, Darwin Hernán., Zhindon, Luis Andres., y Pomaquero, Juan Carlos. (2020). COVID19 y la Educación Virtual Ecuatoriana. Investigación Académica, 1(2), 52-63. Recuperado de https://investigacionacademica.com/index.php/revista/article/view/24

Alonso, Randy., Figueredo, Oscar., Fuentes, Thalía., Izquierdo, Lissett., Garcia, Dianella., y Romeo, Lisandra. (2020). COVID-19 en Cuba: Definiciones del presente curso escolar. Recuperado https://www.google.com/amp/www.cubadebate.cu/noticias/2020/04/29/covid-19-encuba-definiciones-del-presente-curso-escolar-video/amp/

Alonso, Randy., Figueredo, Reinaldo., Fuentes, Thalía., Doimeadiós, Dianet., Izquierdo, Lissett., Romeo, Lisandra., y Fariñas, Lisandra. (2020). Gobierno cubano amplía y extrema medidas para enfrentar la COVID-19. Recuperado de https://www.google.com/amp/www.cubadebate.cu/noticias/2020/03/23/gobiernocubano-amplia-y-refuerza-medidas-para-enfrentar-la-covid-19/amp/

Aquino-Canchari, Christian Renzo., y Medina-Quispe, Camila. (2020). COVID-19 y la educación en estudiantes de medicina. Revista Cubana de Investigaciones Biomédicas, 39(2). Recuperado de http://scielo.sld.cu/scielo.php?script=sci arttext\&pid=S086403002020000200010\&lng=es\&tlng=es

Arce-Peralta, Francisco Javier. (2020). La transición del paradigma educativo hacia nuevos escenarios: COVID-19. CienciAmérica, 9(2). doi: http://dx.doi.org/10.33210/ca.v9i2.285

Arellano, Jesús. (2021). El Aula Virtual como Estrategia Didáctica en un Mundo Transformado por el Covid-19. Revista RedCA, 3(9). doi: https://doi.org/10.36677/redca.v3i9.15823

Areniz-Arevalo, Yesenia., Barrientos-Avendaño, Edwin., Coronel-Rojas, Luis Anderson., Cuesta-Quintero, Fabian., Bayona-lbañez, Eduar., y Rico-Bautista, Dewar. (2020). Plataforma Moodle como apoyo a la presencialidad: Experiencia de uso en la Universidad Francisco de Paula Santander Ocaña en tiempo de Covid-19. Interacción, Revista digital de AIPO, 1(2), 74-82. Recuperado de http://revista.aipo.es/index.php/INTERACCION/article/view/25

Batara, Chris., y Rapat, Charnia Iradat. (2020). Design and implementation of virtual university based on ICT. International Journal of Research-GRANTHAALAYAH, 8(4). doi: https://doi.org/10.29121/granthaalayah.v8.i4.2020.19

Bravo, Julio., Larrea, Carlos., Ruales, Ramiro., y Cruz, Jorge Fernando. (2020). Covid-19: De la educación tradicional y alfabetización de adultos al uso de dispositivos para el interaprendizaje. Brazilian Journal of Health Review, 3(3), 4666-4682. doi: https://doi.org/10.34119/bjhrv3n3-059

Brítez, Mirta. (2020). La educación ante el avance del COVID-19 en Paraguay. Comparativo con países de la Triple Frontera [Preprint]. doi: https://doi.org/10.1590/SciELOPreprints.22 
Cabero-Almenara, Julio., y Llorente-Cejudo, Carmen. (2020). Covid-19: transformación radical de la digitalización en las instituciones universitarias. Campus Virtuales, 9(2), 25-34. Recuperado de http://uajournals.com/ojs/index.php/campusvirtuales/article/view/713

Callís, Sureima., Madrazo, María del Carmen., Guarton, Omara Margarita., Cruz, Virgen., Armas, Ada María de. y Ruiz, Ibis. (2020). El aula virtual en la educación a distancia durante la pandemia de COVID-19. V Congreso virtual de Ciencias Morfológicas. V Jornada Científica de la Cátedra Santiago Ramón y Cajal. Morfovirtual 2020. Recuperado

de http://www.morfovirtual2020.sld.cu/index.php/morfovirtual/morfovirtual2020/paper/view/ $\underline{626 / 556}$

Canales, Roberto. y Silva, Juan. (2020). De lo presencial a lo virtual, un modelo para el uso de la formación en línea en tiempos de Covid-19. Educar em Revista, 36. doi: http://dx.doi.org/10.1590/0104-4060.76140

Cortés, Manuel., Cortés, Manuel., Medina, Juan Felipe., Manzano, Marianelis. y León, Jorge Luis. (2020). Ventajas de la plataforma Moodle para la enseñanza de las Matemáticas en la Universidad de Cienfuegos. Revista Universidad y Sociedad, 12(6), 240-245. Recuperado http://scielo.sld.cu/scielo.php?script=sci arttext\&pid=S221836202020000600240

Del Prete, Annachiara., Cabero, Julio., y Halal, Carol. (2018). Motivos inhibidores del uso del Moodle en docentes de educación superior. Campus Virtuales, 7(2), 69-80. Recuperado de http://www.uajournals.com/ojs/index.php/campusvirtuales/article/view/347/271

Delgado, Ulises., y Martínez, Fernanda. (2021). Entornos virtuales de aprendizaje adoptados en la universidad ante el COVID-19. Diálogos sobre Educación, 12(22), 1-14. doi: https://doi.org/10.32870/dse.v0i22.829

Esquivel, Leidelén., Martínez-Fortún, Maryla., Águila, Madyaret., y Llerena, Elio. (2020). WhatsApps como plataforma para la enseñanza virtual en Imagenología en tiempos de la COVID-19. EduMeCentro, 12(4), 227-234. Recuperado de http://www.revedumecentro.sld.cu/index.php/edumc/article/view/1669

Expósito, Cristián David., y Marsollier, Roxana Graciela. (2020). Virtualidad y educación en tiempos de COVID-19. Un estudio empírico en Argentina. Educación y Humanismo, 22(39), 1-22. doi: https://doi.org/10.17081/eduhum.22.39.4214

Fondevila-Gascón, Joan Francesc., Marqués-Pascual, Joaquín., Mir-Bernal, Pedro., y PoloLópez, Marc. (2019). Usos del WhatsApp en el estudiante universitario español. Pros y contras. Revista Latina de Comunicación Social, 74, 308-324. doi: https://doi.org/10.4185/RLCS-2019-1332

Ghounane, Nadia. (2020). Moodle or Social Networks: What Alternative Refuge is Appropriate to Algerian EFL Students to Learn during Covid-19 Pandemic. Arab World English Journal, 11(3), 21-41. doi: https://dx.doi.org/10.24093/awej/vol11no3.2 
Hernández, Liset. (2020). Precisiones sobre el reinicio del curso académico en la UCLV. Recuperado de https://www.uclv.edu.cu/precisiones-sobre-el-reinicio-del-cursoacademico-en-la-uclv/

Hernández, Yazpik., Raya, Angélica Beatriz., y Santana, María Eugenia. (2017). El WhatsApp como herramienta en el proceso de enseñanza aprendizaje de las humanidades en el nivel superior. Conferencia presentada en IV Congreso Internacional sobre Aprendizaje, Innovación y Competitividad (CINAIC 2017). Zaragoza, España. Recuperado de https://zaguan.unizar.es/record/62958?/n=es

León, Orfelio. y Montero, Ignacio. (2015). Métodos de Investigación en Psicología y Educación. Las tradiciones cuantitativa y cualitativa ( $4^{\mathrm{a}}$ ed.). México: McGraw-Hill Education.

Lovón, Marco Antonio. y Cisneros, Sandra Amelia. (2020). Repercusiones de las clases virtuales en los estudiantes universitarios en el contexto de la cuarentena por COVID19: El caso de la PUCP. Propósitos y Representaciones, 8(3). doi: http://dx.doi.org/10.20511/pyr2020.v8nSPE3.588

Macchiarola, Viviana., Pizzolitto, Ana Lucía., Pugliese, Verónica., y Muñoz, Diego José. (2020). La enseñanza con modalidad virtual en tiempos del covid19. La mirada de los estudiantes de la Universidad Nacional de Río Cuarto. Contextos de Educación, 28(20), 1-13. $\quad$ Recuperado http://www2.hum.unrc.edu.ar/ojs/index.php/contextos/article/view/1086/1148

Mezarina, Jhon., Evaristo, Teresa., Ortiz, María., y Usca, Stefany. (2020). Factibilidad de las clases virtuales en la Facultad de Odontología de la Universidad Nacional Mayor de San Marcos en tiempos de COVID-19. Revista Odontológica Basadrina, 4(2), 10-17. doi: https://doi.org/10.33326/26644649.2020.4.2.957

Ordoñez, Klondy Giovanna. (2020). La construcción de la comunicación interpersonal educativa, mediada por redes sociales y plataformas educativas, en las carreras de Ciencias de la Educación y Psicología, de la Universidad Mayor de San Andrés. Educación Superior, 7(1), 80-92. Recuperado de http://ojs.cepies.umsa.bo/index.php/RCV/article/view/63/0

Pérez-Jorge, David., Gutiérrez-Barroso, Josué., Castro-León, Fátima., Rodríguez-Jiménez, María del Carmen., Márquez-Domínguez, Yolanda., y González, Ana Isabel. (2018). Herramienta síncrona de comunicación para la mejora del asesoramiento, seguimiento y tutorización del alumnado universitario: la experiencia del uso del WhatsApp. 370-378. En A. Vega y D. Stendardi (Coords. ), De la innovación imaginada a los procesos de cambio (pp- 369-378). Recuperado de https://dialnet.unirioja.es/servlet/articulo?codigo $=6509304$

Pérez-López, Eva., Vázquez, Alfonso., y Cambero, Santiago. (2020). Educación a distancia en tiempos de COVID-19: Análisis desde la perspectiva de los estudiantes universitarios. RIED: Revista Iberoamericana de Educación a Distancia, 24(1), 331-350. doi: http://dx.doi.org/10.5944/ried.24.1.27855 
Sobaih, Abu Elnasr., Hasanein, Ahmed., y Abu, Ahmed. (2020). Responses to COVID-19 in Higher Education: Social Media Usage for Sustaining Formal Academic Communication in Developing Countries. Sustainability, 12(16). doi: https://doi.org/10.3390/su12166520

Sol, Indira. (2021). Evalúa MES inicio y desarrollo del curso escolar 2021. Recuperado de https://www.uclv.edu.cu/evalua-mes-inicio-y-desarrollo-del-curso-escolar-2021/

Suárez, Belén. (2018). Whatsapp: su uso educativo, ventajas y desventajas. Revista de Investigación en Educación, 16(2), 121-135. Recuperado de http://reined.webs4.uvigo.es/index.php/reined/article/view/342/386

Susilawati, Samsul. y Supriyatno, Triyo. (2020). Online Learning Through WhatsApp Group in Improving Learning Motivation in the Era and Post Pandemic COVID -19. Jurnal Pendidikan, 5(6), 852-859. Recuperado de https://www.google.com/url?sa=t\&source=web\&rct=j\&url=http://repository.uinmalang.ac.id/6923/1/6923\%2520jurnal\%2520UM\%25202020\%252013670-20567-1SM.pdf\&ved=2ahUKEwio77Hk75rvAhXBt1kKHcGTAeAQFjAKegQICRAC\&usg=AOvVa w1C8gxKcP6LFfKaa94RYAWV

Torres, Carlos Arturo. (2018). Formas de participación en línea en estudiantes de la Facultad de Administración de la Universidad Veracruzana en México. Actualidades Investigativas en Educación, 18(2). doi: https://doi.org/10.15517/aie.v18i2.33131

Trejos, Omar Iván. (2018). WhatsApp como herramienta de apoyo al proceso de enseñanza y aprendizaje de la programación de computadores. Revista Educación y Ciudad, (35), 149-158. Recuperado de https://revistas.idep.edu.co/index.php/educacion-yciudad/article/view/1970

Universidad Central "Marta Abreu" de Las Villas (UCLV). (2021). Opciones para el uso de las plataformas virtuales. Recuperado de www.uclv.edu.cu

UNESCO. (2020). El Secretario General de las Naciones Unidas advierte de que se avecina una catástrofe en la educación y cita la previsión de la UNESCO de que 24 millones de alumnos podrían abandonar los estudios. Recuperado de https://es.unesco.org/news/secretario-general-naciones-unidas-advierte-queseavecina-catastrofe-educacion-y-cita

Vega, Leudis Orlando., Pupo, Aylín., Tapia, Ileana Irene., y Pérez, Marian. (2021). Defensa de tesis de diploma vía "Online" como alternativa en situaciones excepcionales de COVID19. Revista Estudios del Desarrollo Social: Cuba y América Latina, 9(1), 233-243. Recuperado de http://scielo.sld.cu/scielo.php?script=sci arttext\&pid=S2308$\underline{01322021000100004}$

Yudha, Fietyata., Luthfi, Ahmad., y Prayudi, Yudi. (2017). A proposed model for investigating on web WhatsApp application. Advanced Science Letters, 23(5), 4050-4054. doi: https://doi.org/10.1166/asl.2017.8308 


\section{Anexo 1: Encuesta sobre empleo de plataformas virtuales}

Estimado estudiante:

Durante la actual situación epidemiológica a raíz de la COVID-19 en nuestro país, se ha recurrido al empleo de plataformas virtuales para la comunicación y el intercambio en materia de proceso docente. La presente encuesta pretende indagar acerca del uso de dichas plataformas por parte del alumnado universitario. Le solicitamos responda con la mayor sinceridad posible. La encuesta es completamente anónima, y los datos ofrecidos serán utilizados con fines investigativos.

Gracias por su colaboración.

\section{Datos sociodemográficos:}

Edad

Año que cursa

Zona de residencia

Sexo

Provincia de residencia

\section{Interrogantes:}

1. ¿Qué plataformas emplea para la resolución de sus tareas académicas? (Selección múlitple)

$\begin{array}{llll}\text { _WhatsApp } & \text { - Moodle } & \text {-Correo UCLV } & \text { N Nube personal } \\ \text { _Telegram } & \text { _Sijú } & \text { _Correo personal } & \text { _Nube UCLV }\end{array}$

2. Ordene las plataformas seleccionadas por usted en la pregunta anterior de modo tal que, en primer lugar, coloque la que más utiliza, y al final, la que menos emplea.

3. ¿Por qué la plataforma ubicada en primer lugar, es su preferida? ( $0=$ no; $1=s i ́)$
a) Facilita la comunicación.
b) Resulta muy frecuente su uso entre mis colegas de estudio, por lo que hay más posibilidades de que estén en línea cuando los necesito.
c) Me encuentro familiarizado (a) con su funcionamiento.
d) Me resulta atractiva.

4. ¿Por qué la plataforma ubicada al final, es la menos preferida por Ud.?
a) No resulta de fácil acceso.
b) Se demora en cargar; es lenta.
c) Se dificulta el envío de archivos grandes y/o archivos múltiples.
d) La mayoría de las personas con quienes comparto para tareas académicas, no la usan.
e) No me encuentro familiarizado (a) con su funcionamiento.
f) No me atrae. 
Revista indizada en

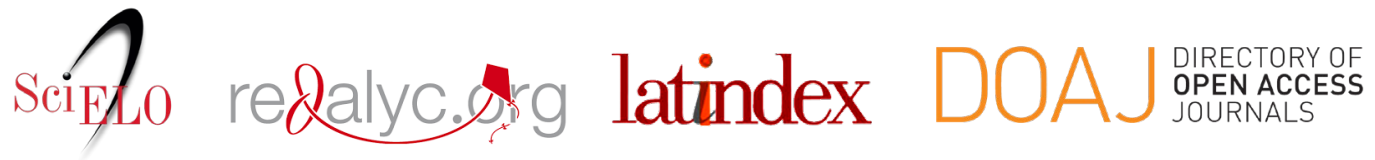

Distribuida en las bases de datos:

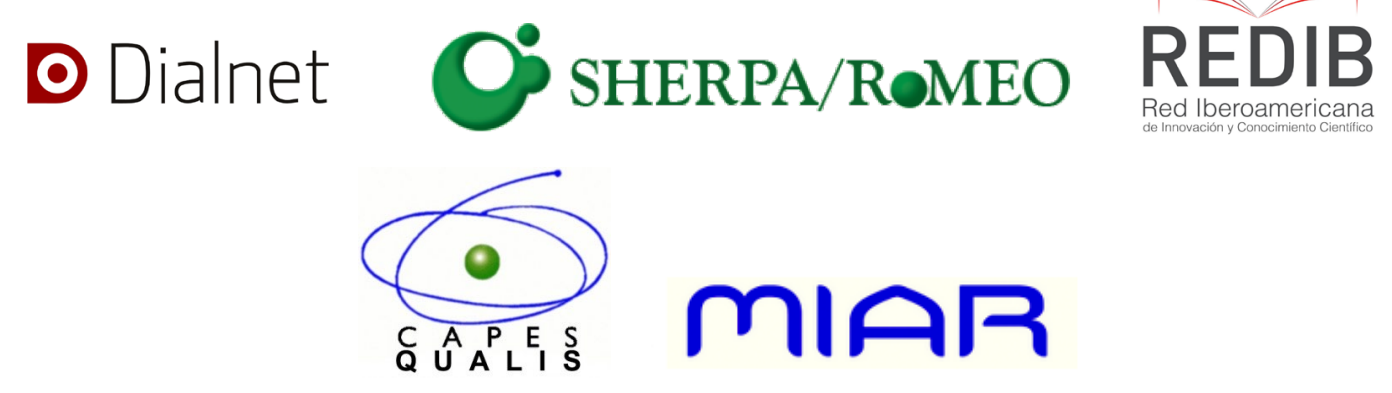

